\title{
Electrochemotherapy for Pancreatic Cancer: An Emerging Treatment Modality?
}

\author{
Jin He, MD, PhD, FACS \\ Department of Surgery, The Johns Hopkins University School of Medicine, Baltimore, MD
}

Electrochemotherapy is defined as using electroporation to improve the delivery of chemotherapy drugs into cells. ${ }^{1}$ Since the 1990s, electrochemotherapy of cutaneous melanoma with bleomycin and cisplatin has achieved an objective response rate of $85 \% .^{2}$ However, its application to intra-abdominal malignancy has only been tested in the liver. ${ }^{3}$ Electrochemotherapy in pancreatic adenocarcinoma has not been well established. ${ }^{4}$ Because of delayed diagnosis and early recurrence, pancreatic adenocarcinoma has been generally considered as a systemic disease. ${ }^{5}$ Local treatments alone, such as aggressive surgical resection or radiation therapy, can only help some patients with localized pancreatic adenocarcinoma. Potent and effective chemotherapies such as fluorouracil, leucovorin, irinotecan, and oxaliplatin (FOLFIRINOX) or gemcitabine/nabpaclitaxel have resulted in improved systemic control and changed the treatment paradigm. ${ }^{6}$ With improved systemic control, there has been a cascade of publications on aggressive local control of advanced pancreatic adenocarcinoma after chemotherapy. ${ }^{7}$

In the article that accompanies this editorial, ${ }^{8}$ Martin et al. published their preliminary experience on a novel electrochemotherapy approach using irreversible electroporation (IRE) and modern chemotherapy regimens, including gemcitabine/nab-paclitaxel or FOLFIRINOX. The group led by Martin et al. has pioneered the application of IRE in the treatment of advanced pancreatic adenocarcinoma. ${ }^{9}$ Over the last decade, Martin's group and others have shown the feasibility and effectiveness of IRE

(C) Society of Surgical Oncology 2020

First Received: 5 June 2020;

Published Online: 4 July 2020

J. He, MD, PhD, FACS

e-mail: jhe11@jhmi.edu for patients with locally advanced pancreatic cancer. A novel electrochemotherapy approach using IRE and active chemotherapy agents can improve drug delivery to the complex tumor microenvironment of pancreatic cancer. Using the orthotopic mouse model, Martin et al. showed this electrochemotherapy approach had a synergistic treatment effect via the apoptosis pathway and anti-proliferation mechanism. What is more intriguing than the results from the mouse model is the safety and efficacy outcome from five patients with stage three pancreatic cancer treated with this electrochemotherapy approach. They observed no toxicity or disease progression at a median follow-up of 81 days after the surgery.

Martin's group is to be commended for this well-designed comprehensive preclinical study. This novel electrochemotherapy approach has great potential in helping patients with advanced pancreatic cancer. Like many other exciting studies, more information is needed to fill in certain knowledge gaps. First, data from prospective studies on electrochemotherapy are urgently needed to help patients with pancreatic cancer. It is exciting to know that Martin's group has started a prospective dose-escalating safety and tolerability study of chemotherapy and IRE in the treatment of advanced pancreatic adenocarcinoma (NCT03484299). All patients undergoing IRE by experienced surgeons will receive chemotherapy with FOLFIRINOX or gemcitabine. This prospective study will lay the foundation for future randomized efficacy studies. Second, many high-volume centers of pancreatic surgery don't have the proper setup or adequate experience to perform IRE. Being able to educate other pancreas surgeons to master IRE will be the key to conducting multicenter prospective studies of electrochemotherapy in the future. Finally, the tumor microenvironment in patients with pancreatic cancer is complex. Whether the electrochemotherapy approach using IRE and FOLFIRINOX 
can improve the tumor antigen presentation or affect tumor immunogenicity is unknown. With the success of immune checkpoint inhibitors in many other solid tumors, the potential of combining electrochemotherapy and immune checkpoint inhibitors remains to be defined.

DISCLOSURES The author declares no conflict of interest.

\section{REFERENCES}

1. Jaroszeski MJ, Gilbert R, Heller R. Electrochemotherapy: an emerging drug delivery method for the treatment of cancer. $A d v$ Drug Deliv Rev. 1997;26:185-197.

2. Marty M, Sersa G, Garbay JR, Gehl J, Collins CG, Snoj M, et al. Electrochemotherapy-an easy, highly effective and safe treatment of cutaneous and subcutaneous metastases: results of ESOPE (European Standard Operating Procedures of Electrochemotherapy) study. Eur J Cancer Suppl. 2006;4:3-13.

3. Zmuc J, Gasljevic G, Sersa G, Edhemovic I, Boc N, Seliskar A, et al. Large liver blood vessels and bile ducts are not damaged by electrochemotherapy with bleomycin in pigs. Sci Rep. 2019;9:1-11.

4. Granata V, Fusco R, Piccirillo M, Palaia R, Petrillo A, Lastoria S, et al. Electrochemotherapy in locally advanced pancreatic cancer: preliminary results. Int J Surg. 2015;18:230-236.
5. Groot VP, Rezaee N, Wu W, Cameron JL, Fishman EK, Hruban $\mathrm{RH}$, et al. Patterns, timing, and predictors of recurrence following pancreatectomy for pancreatic ductal adenocarcinoma. Ann Surg. 2018;267:936-945.

6. Conroy T, Hammel P, Hebbar M, Ben Abdelghani M, Wei AC, Raoul JL, et al. FOLFIRINOX or gemcitabine as adjuvant therapy for pancreatic cancer. $N$ Engl J Med. 2018;379:2395-2406.

7. Bachellier P, Addeo P, Faitot F, Nappo G, Dufour P. Pancreatectomy with arterial resection for pancreatic adenocarcinoma: How can it be done safely and with which outcomes?: A single institution's experience with 118 patients. Ann Surg. 2020;271:932-940.

8. Bhutiani N, Li Y, Zheng Q, et al. Electrochemotherapy with irreversible electroporation and FOLFIRINOX improves survival in murine models of pancreatic adenocarcinoma. Ann Surg Oncol. 2020. https://doi.org/10.1245/s10434-020-08782-2.

9. Martin RC, Kwon D, Chalikonda S, Sellers M, Kotz E, Scoggins C, et al. Treatment of 200 locally advanced (stage III) pancreatic adenocarcinoma patients with irreversible electroporation: safety and efficacy. Ann Surg. 2015;262:486-494 (discussion 492-494).

Publisher's Note Springer Nature remains neutral with regard to jurisdictional claims in published maps and institutional affiliations. 